\title{
Perfiles de drogodependientes en relación con variables y trastornos de personalidad
}

\section{Profiles of drug addicts in relation to personality variables and disorders}

\author{
María Carou*; Estrella Romero**; Mán Ángeles Luengo** \\ *Unidad Municipal de Atención a Drogodependientes (UMAD), Ayuntamiento de Santiago de Compostela (Municipal Centre \\ for Attention to Drug Addicts, Santiago de Compostela City Council); **Facultad de Psicología, Universidad de Santiago de \\ Compostela, USC (Psychology Faculty, University of Santiago de Compostela)
}

\section{Resumen}

En las últimas décadas, la investigación ha permitido identificar un conjunto de variables de personalidad impulsiva/desinhibida estrechamente asociadas a la adicción a drogas. Así mismo trastornos vinculados a estas variables, como el TDAH y los trastornos de personalidad, están siendo objeto de vigorosas líneas de trabajo en el ámbito de las drogodependencias. A pesar de que se ha acumulado mucho conocimiento sobre la relación de estas variables y trastornos, tomados aisladamente, se sabe menos acerca de cómo estos constructos permiten identificar perfiles específicos dentro de la población de drogodependientes. Este trabajo, partiendo de los datos recogidos en una muestra de drogodependientes a tratamiento, analiza cómo la impulsividad, la búsqueda de sensaciones, el autocontrol, el TDAH y los trastornos de personalidad permiten identificar tipos específicos de adictos. El análisis cluster permitió delimitar dos perfiles atendiendo a estas características de personalidad y psicopatológicas, destacando como variables que contribuyen a esta diferencia el autocontrol y la impulsividad, los trastornos de personalidad impulsivo y disocial, así como las puntuaciones en TDAH. Uno de esos perfiles (un 56.1\% de los participantes) con un patrón personal de alta desinhibición, se asocia con indicadores de consumo y criminológicos de especial severidad. Estos resultados permiten subrayar el papel de la personalidad y de los trastornos asociados a la impulsividad en la identificación de perfiles distintivos dentro de la población de adictos, y sugieren la necesidad de generar estrategias de tratamiento ajustadas a las configuraciones personales/psicopatológicas de los drogodependientes.

Palabras clave: Personalidad; Impulsividad; TDAH; Trastornos de la Personalidad; Adicción.

\begin{abstract}
In recent decades, research has identified a set of impulsive/ disinhibited personality variables closely associated with drug addiction. As well as this, disorders linked with these variables, such as ADHD and personality disorders, are being closely studied in the field of drug addiction. Although much knowledge has been accumulated about the relation of these variables and disorders taken separately, less is known about how these constructs allow identify-specific profiles within the drug dependent population to be identified. This work, on the basis of data collected on a sample of drug addicts in treatment, analyzes how impulsiveness, sensation seeking, self-control, ADHD and personality disorders contribute to identifying specific profiles of addicts. Cluster analysis allowed two profiles to be outlined according to these personality and psychopathology characteristics. Self-control, impulsiveness, impulsive and antisocial personality disorders, as well as scores in ADHD, emerge as the variables that contribute more to profile differentiation. One of these profiles ( $56.1 \%$ of participants) with a high disinhibition pattern, is associated with severe indicators of consumption and criminal career patterns. These results allow us to emphasize the role of personality and impulsiveness-related disorders in the identification of distinctive profiles within the addict population, and suggest the need to generate treatment strategies adapted to personal/psychopathology configurations of drug addicts. Keywords: Personality; Impulsivity; ADHD; Personality Disorders; Addiction
\end{abstract}


$\mathrm{E}$ 1 auge en los últimos años de las investigaciones en el ámbito de los procesos psicobiológicos subyacentes al proceso de adicción, ha puesto de relieve la importancia de variables de personalidad de carácter temperamental como la impulsividad o la búsqueda de sensaciones.

La impulsividad ha sido relacionada con el consumo de drogas y con otras conductas problemáticas en múltiples estudios, observándose en adictos, menor capacidad de inhibición de respuesta, dificultades para la reflexión y la planificación y tendencia a elegir reforzadores menores y más cercanos en tareas de demora de la gratificación (Olmstead, 2006), así como a presentar conductas violentas, pobre regulación comportamental y menor empatía (Romero-Martínez y Moya-Albiol, 2015). La relación entre impulsividad y abuso de sustancias muestra una relación robusta, a lo largo de muy diferentes estudios, identificándose como una de las variables más consistentemente vinculadas tanto al contacto temprano con sustancias como a la progresión en la adicción (Belin, Mar, Dalley, Robbins y Everitt, 2008; Gullo, Loxton y Dawe, 2014; Motos-Sellés, Cortés-Tomás, Giménez-Costa, y Cadaveira-Mahía, 2015; Stauzt y Cooper, 2013).

Dentro del estilo de personalidad orientada a la acción característica de las personas drogodependientes, otra variable relevante es la búsqueda de sensaciones (Zuckerman, 1979). La búsqueda de sensaciones se considera un predictor importante del inicio del consumo de drogas en la adolescencia (Luengo, Otero-López, Romero y Gómez, 1996), y al igual que la impulsividad, se ha relacionado con dificultades para el control inhibitorio (Fillmore, Ostling, Martin y Kelly, 2009) y con mayor sensibilidad a efectos de las drogas (Nadal-Alemany, 2008).

El autocontrol es un constructo que aglutina diferentes variables como impulsividad, demora de gratificación y toma de riesgo entre otros (Romero, Gómez-Fraguela, Luengo y Sobral, 2003). El autocontrol ha adquirido especial relevancia en los últimos años en el campo de la conducta desviada y el consumo de drogas, a partir de los planteamientos de Gottfredson y Hirschi (1994), y destaca como uno de los correlatos más consistentes de las conductas que transgreden normas (Romero, Sobral, y Luengo, 1999), incluyendo problemas relacionados con las drogas (Gallupe y Baron, 2014; Romero et al., 2003) y cronicidad en el consumo (López-Torrecillas, Peralta, Muñoz-Rivas y Godoy, 2003).

Del mismo modo que dimensiones como impulsividad, búsqueda de sensaciones o autocontrol se han visto implicadas en el consumo de drogas, los estudios sobre personalidad y trastornos han proporcionado un cuerpo de conocimiento de indudable valor para entender las adicciones. En los últimos años se ha prestado considerable atención a entidades psicopatológicas estrechamente relacionadas con estos aspectos de la personalidad, específicamente el Trastorno por Déficit de Atención con Hiperactividad (TDAH) y los Trastornos de la Personalidad (TP) son un activo centro de interés dentro del campo de las adicciones.

Así, el TDAH, estrechamente relacionado con la impulsividad y con el ámbito de la personalidad desinhibida, recibe cada vez más atención en el estudio del consumo de drogas (e.g., Wilens, 2007). Aunque el TDAH se diagnostica principalmente en niños, se estudia cada vez más su relevancia en los adultos (e.g., Grogan y Bramham, 2016); en algunos estudios se ha mostrado que con la edad, se atenúan los síntomas relacionados con la hiperactividad y tienen mayor estabilidad la inatención y la impulsividad, y que los síntomas persistentes a partir de la adolescencia se asocian a especiales dificultades clínicas y psicosociales (Romero y Alonso, 2015), incluyendo trastornos relacionados con la agresividad, conductas antisociales, conductas de riesgo, accidentes de tráfico y dificultades para administrar tareas cotidianas, así como con comorbilidad psiquiátrica (Ramos, Bosch, Castells, Nogueira, García y Casas, 2006). En cuanto al consumo de drogas, se ha informado que, en adictos, la prevalencia del TDAH es alta (Kalbag y Levin, 2005), agravando la severidad de ambos trastornos: TDAH y adicción. Otros estudios se han centrado en las tasas de adicción en grupos de adultos con y sin TDAH, y los resultados muestran también un porcentaje significativamente mayor de adictos entre los afectados por TDAH (Biederman, Wilens, Mick, Milberger, Spencer y Faraone, 1995). Se ha constatado que el TDAH puede afectar el curso de abuso de sustancias, prediciendo la edad más temprana de comienzo, la duración más larga de la adicción, la progresión en la adicción y el fracaso del tratamiento (Schubiner et al, 2000).

Los TP constituyen también un campo cuya investigación en relación con las adicciones ha crecido exponencialmente en los últimos años (Magnavita, 2004). Así, por ejemplo, en un metaanálisis sobre 16 estudios de comorbilidad de trastornos psiquiátricos en adictos a opiáceos, se encuentra una prevalencia del 42\% (Frei y Rehm, 2002) en esta población. En adictos a la cocaína, se han encontrado cifras de presencia de TP de entre 47\% y 97\% (López y Becoña, 2006). Otros estudios han mostrado que, en general, en adictos a tratamiento, la prevalencia de TP oscila entre el 44,3\% (Echeburúa, de Medina y Aizpiri, 2007) y el 35-73\% (Verheul, 2001). Específicamente, la investigación ha mostrado que en los consumidores de sustancias, el TP antisocial (con cifras entre el 18-30\%) y el trastorno límite (7-22\%) son los más frecuentes (Verheul, van der Brink y Hartgers 1995). También se ha demostrado que la presencia de los TP se relaciona con mayor severidad de la adicción y con una mayor probabilidad de interrupciones en el tratamiento (González, 2014).

En general, estas diferentes líneas de investigación apuntan a la importancia de la impulsividad y de las dimensiones y trastornos asociados a ella en la comprensión del consumo de drogas. La investigación previa permite concluir que tanto la personalidad impulsiva como los trastornos relacionados con la desinhibición y la falta de autocontrol son ingredientes clave en la caracterización psicológica de los adictos. 
Otra área de interés en el estudio de las adicciones y que merece más desarrollo es la elaboración de tipologías dentro de la población de adictos que permitan entender mejor los procesos que llevan a la adicción y adecuar las estrategias de tratamiento.

A partir de técnicas de análisis cluster, Cloninger (1987) identifica dos subtipos de consumidores de alcohol que se diferencian en la edad de inicio en el consumo, la heredabilidad de la adicción, factores de riesgo en la niñez, grado de severidad de la dependencia y desajuste psicosocial. Estas tipologías se corresponderían a otras encontradas por Babor et al. (1992) y Ball (1995). En nuestro país en un estudio con policonsumidores sometidos a tratamiento (Muñoz, Nava, Graña y Martínez, 2006), se identifican dos grupos que presentan diferencias significativas en variables sociodemográficas, variables de consumo y severidad en la adicción: un grupo de consumidores funcionales (tipo A), compuesto por consumidores de cocaína, con menos problemas médicos, psicosociales y psicopatológicos, y un grupo de consumidores cronificados (tipo B), compuesto por consumidores de heroína y politoxicómanos, con un mayor deterioro funcional y social, y con más infracciones legales. A partir de la clasificación en estos dos grupos de consumidores, en un estudio posterior (Graña, Muñoz y Navas, 2009) se examinaron las diferencias en variables y trastornos de personalidad entre los dos grupos; los resultados demostraron que, mientras los consumidores funcionales tenían significativamente puntuaciones mas altas en extraversión y cordialidad, los consumidores crónicos presentaban puntuaciones más altas en TP esquizoide y disocial.

Teniendo en cuenta estos resultados, en el presente estudio se pretende avanzar en la diferenciación de perfiles de drogodependientes a partir de variables y trastornos de personalidad que en la investigación parecen tener relevancia en el proceso de la adicción. En un estudio previo (Carou, Romero, Luengo, 2013) en el que se analizaban variables de personalidad y patrones de consumo en drogodependientes a tratamiento, se planteaba la necesidad de tomar en consideración un grupo especial de adictos a los que se denominó "cocaheroinómanos", formado por pacientes heroinómanos rehabilitados y que años después desarrollan una adicción a cocaína. Este grupo presentaba un perfil de mayor gravedad de la adicción y la marginalidad y peculiaridades en las variables de personalidad analizadas.

En el presente trabajo, con un enfoque más centrado en la persona (Bergman y Magnusson, 1997) que en las variables, se pretende comprender la heterogeneidad de los adictos y analizar si se pueden identificar perfiles distintivos atendiendo específicamente a las variables de personalidad y a los trastornos que, como se ha analizado anteriormente, parecen relevantes para el consumo. El análisis de perfiles de adictos basados en sus características de personalidad permitirá profundizar en los procesos que llevan a la adicción y en el desarrollo de tratamientos ajustados a las pecu- liaridades personales y funcionales de los drogodependientes. La necesidad de identificar tipos específicos de adictos ha sido recalcada en la literatura previa, y el amplio cuerpo de investigación generado en la última década en torno a la personalidad y sus trastornos, así como al TDAH, sugiere la conveniencia de atender a estos constructos para deslindar perfiles específicos de consumidores.

Así pues, el objetivo principal planteado por el presente estudio es identificar perfiles específicos de adictos en relación con sus variables de personalidad y trastornos (TP y TDAH). Como objetivos específicos se pretende a) determinar qué variables y trastornos presentan un mayor peso para diferenciar perfiles de adictos, b) examinar cómo estos perfiles se asocian con la pertenencia a distintos grupos de consumidores; c) analizar si tales perfiles se relacionan con indicadores de gravedad en el consumo y características criminológicas.

Como hemos señalado, diferentes investigaciones previas sugieren la especificación de dos patrones diferenciados, y por ello es esperable que, atendiendo ahora a variables y trastornos de personalidad, estos dos perfiles puedan ser identificados en una muestra de adictos a tratamiento.

\section{Método}

\section{Participantes}

Para la realización del presente estudio fueron evaluados 176 adultos adictos, a tratamiento en una Unidad Asistencial de Drogodependencias (UAD) de la red pública de Galicia, España. De ellos, un $47.2 \%$ presentaron diagnóstico de dependencia a heroína, y un $52.8 \%$ a cocaína. De los adictos a la cocaína, 16 participantes eran cocainómanos con adicción previa a la heroína. Dado que los estudios previos (Carou et al., 2013) mostraron que estos cocaheroinómanos presentan peculiaridades significativas de corte personal y psicosocial, en el presente trabajo se tienen en cuenta estos tres tipos de consumidores: heroína, cocaína y cocaheroína.

Los principales criterios de inclusión en la muestra fueron: dependencia a cocaína o heroína según criterios CIE10 , tener más de 18 años y firmar el consentimiento informado. Se descartan pacientes a tratamiento con dependencia a otra sustancia y también pacientes que presentan consumo problemático pero sin presentar dependencia.

\section{Instrumentos}

GECEAS (Gestión de Centros Asistenciales). Se trata de una aplicación informática a través de la cual se elabora una base de datos recogidos en entrevistas con el paciente. Además de sus funciones de recogida de información y evaluación, funciona como aplicación de gestión clínica para los centros de drogas en Galicia y permite manejar integralmente todos los procesos que tienen lugar en una UAD, cubriendo los ámbitos clínico, asistencial y de gestión. En este estudio se utiliza su Módulo de Historia Clínica, que permite codificar variables sociodemográficas, de consumo 
(e.g., edad de inicio, frecuencia de consumo), de tratamiento (e.g., tratamientos previos) y criminológicas (e.g., número de detenciones, meses en prisión).

Escala de Impulsividad de Barratt, versión 11 (BIS-11; Patton, Stanford y Barratt, 1995; adaptación española de Oquendo et al., 2001). Se trata de una escala autoaplicada para evaluar la impulsividad, compuesta por 30 ítems que se responden en una escala tipo Likert de 4 puntos, proporcionando puntuaciones en 3 dimensiones de la impulsividad (motora, cognitiva y no planificación), cuya suma aporta una medida de impulsividad total. Para el presente estudio se utilizó la puntuación total, con una consistencia interna (alpha de Cronbach) de .79. Propiedades psicométricas adecuadas, en cuanto a fiabilidad y validez, fueron encontradas también en estudios previos con esta escala (Carrillo-de-la-Peña, Otero y Romero, 1993; Oquendo et al., 2001).

Escala de Búsqueda de Sensaciones, forma V, o SSS-V (Zuckerman, Eysenck y Eysenck, 1978; adaptación española de Pérez y Torrubia, 1986). Está compuesta por 40 ítems de elección forzada, que permiten obtener puntuaciones en cuatro subescalas (búsqueda de emociones y aventuras, búsqueda de experiencias, desinhibición y susceptibilidad al aburrimiento) y también una puntuación total que resulta de la suma de las cuatro subescalas; esta puntuación total fue la utilizada en el presente trabajo. La fiabilidad de esta escala en este estudio (alpha de Cronbach) fue de .76, e índices semejantes se han encontrado en otros trabajos (Romero, Luengo y Sobral, 2001).

Escala de Autocontrol de Grasmick, Title, Bursik y Arneklev (1993; adaptación española de Romero et al., 2003). Esta escala contiene 24 ítems, con respuesta dicotómica, que permiten obtener una puntuación total en autocontrol de acuerdo con el modelo de Gottfredson y Hirschi (1990). La escala ha mostrado su utilidad psicométrica para evaluar el constructo autocontrol en estudios previos (e.g., Romero et al., 2003); en este estudio la medida global presentó, de forma semejante a la adaptación española previa, una consistencia interna (alpha de Crombach) de .89.

Cuestionario Autoinformado de Cribado de TDAH en el Adulto ASRS-V1.1 (Adult ADHD Self-Report Scale) desarrollada entre la OMS y el grupo de trabajo sobre adultos con TDAH de Adler, Kessler, Spencer en 2005 (Kessler et al., 2005), sigue criterios del DSM-IV, y se centra en sintomatología actual de TDAH en adultos. En este estudio se utilizó la versión reducida de cribado de 6 ítems (en adaptación española de Daigre et al., 2009), y que constituye el único cuestionario de cribado validado en español y con adecuadas garantías psicométricas para TDAH en adultos. Aunque la escala permite obtener una categorización dicotómica entre "probable" y "no probable", también permite utilizar puntuaciones dimensionales ( 0 a 24$)$, resultantes de sumar las puntuaciones (0 a 4; de "nunca" a "muy frecuentemente") de cada ítem (Kessler, Adler, Gruber, Sarawate, Spencer y Van Brunt, 2007). Esta puntuación di- mensional fue la utilizada en el presente estudio, con una fiabilidad alpha de Crombach de .65 .

Cuestionario de evaluación asociado a la International Personality Disorder Examination (IPDE, Loranger, 1994), adaptado en España por López-Ibor, Pérez-Urdaniz, y Rubio-Larrosa (1996). La IPDE es un instrumento ampliamente utilizado para proporcionar puntuaciones en nueve TP identificados por la OMS. Contiene 59 ítems a los que el individuo responde "Verdadero" o "Falso", describiendo su comportamiento durante los últimos 5 años. En esta investigación se utiliza el cuestionario de screening, y se utilizaron, para los análisis estadísticos, las puntuaciones dimensionales obtenidas correspondientes a cada uno de los trastornos, con alphas de Crombach entre .43 (TP paranoide) y .63 (TP ansioso), que son semejantes a las que se han informado en estudios previos (Slade y Forrester, 2013).

\section{Procedimiento}

Los pacientes que acuden a la UAD buscan tratamiento voluntario para su drogodependencia, y en su admisión o readmisión a tratamiento entran en el programa de orientación y acogida, se toman los datos necesarios para cumplimentar su Historia Clínica informatizada a través del programa GECEAS, y son diagnosticados por especialistas en psicología clínica y psiquiatría, a través de entrevista clínica. Para el presente estudio además se realizó la cumplimentación de los instrumentos autoinformados previamente mencionados. La cumplimentación tuvo una duración media de 60 minutos, y fue realizada en las dependencias del centro, individualmente, garantizando la confidencialidad de los datos proporcionados y bajo la supervisión de la primera autora de este trabajo. La realización del estudio fue aprobada por la dirección del dispositivo, y se garantizó la adecuación a los principios éticos pertinentes; así, el proyecto se guió por los principios recogidos en la Declaración de Helsinki, respetando escrupulosamente los derechos de los participantes al inicio y durante el transcurso del estudio.

\section{Análisis estadísticos}

En primer lugar, se calcularon los estadísticos en los principales descriptores de la muestra (sociodemográficos, de consumo, criminológicos), así como las correlaciones de Pearson entre las escalas (de personalidad y trastornos) aplicadas en este estudio. Para la identificación de perfiles atendiendo a las variables de personalidad y trastornos se utilizó un análisis cluster con un algoritmo bietápico, que realiza una preagrupación (preclustering) y una posterior agrupación jerárquica. La medida de distancia considerada fue la euclídea, y para determinar el número óptimo de clusters se tuvo en cuenta el criterio bayesiano de Schwartz (BIC). Para la caracterización de los clusters resultantes y para analizar su relación con variables de consumo y criminológicas se utilizaron tablas de contingencia con chi-cuadrado como estadístico de contraste, y análisis multivariables de la varian- 
za (MANOVA), incorporando la corrección de Bonferroni para minimizar el error tipo I. Para cada uno de los análisis realizados en este estudio se consideraron los participantes que presentaban datos válidos en todas las variables incluidas en el análisis. Los análisis fueron realizados a través del paquete estadístico IBM SPSS 20.

\section{Resultados}

\section{Análisis preliminares: descriptivos y correlaciones entre las variables}

En primer lugar, y respecto a los descriptivos de la muestra (véase Tabla 1), el $76.7 \%$ de los participantes eran varones y el $23.3 \%$ mujeres. La edad media fue de 32.1 años. La mayoría $(55.7 \%)$ convivía con la familia de origen, estaban solteros $(74.4 \%)$ y sin hijos $(71 \%)$. En cuanto a los ingresos referidos a los últimos 6 meses anteriores al inicio de tratamiento, la mitad de la muestra trabajaba y la otra mitad recibía ayudas (familiares, sociales) o subsistía a partir de actividades marginales.

En cuanto a las características del consumo, la edad media de inicio en el consumo de la droga principal por la que acudían a tratamiento fue de 19.4 años. Al inicio del tratamiento la mayoría $(60.2 \%)$ presentaba un consumo diario. Destaca el alcohol como sustancia problemática co-principal en un $44.3 \%$, seguida de cocaína (en heroinómanos) y cannabis, ambos con un $22.2 \%$. Además hay otras sustancias consumidas de manera habitual, entre las que destaca el tabaco para la práctica totalidad de la muestra (94.3\%), el alcohol (para el $77.8 \%$ ), y el cannabis (para el $62.5 \%$ ). Cabe destacar una media de 0.8 urgencias hospitalarias debidas al consumo, aunque el $62.5 \%$ nunca las ha tenido. Para el $59.7 \%$ no es el primer tratamiento al que acuden para un problema adictivo, y además el $48.3 \%$ ha tenido tratamientos psiquiátricos anteriores.

Con respecto a las variables criminológicas informadas, el $34.7 \%$ han tenido problemas legales, presentan una media de edad en la primera detención de 23.3 años, una media de 3.1 detenciones, 3.7 meses en prisión y 1.1 procesos judiciales cumplidos.

En segundo lugar, y con el fin de determinar la relación que se establece entre las medidas de personalidad y trastornos tomadas en este estudio, se realizaron análisis de correlación de Pearson (ver Tabla 2).

Cuando se examina cómo correlacionan entre sí las variables de personalidad se observa que todas ellas presentan correlaciones significativas. Los trastornos entre sí también presentan asociaciones significativas; las correlaciones más altas son las que se establecen entre el TP impulsivo con el TP disocial y el TDAH, y entre el TP dependiente con los TP ansioso y límite.

En cuanto a las correlaciones entre dimensiones de personalidad y trastornos, las correlaciones más altas para la impulsividad se establecen con el TDAH y el TP impulsivo; la
Tabla 1. Perfil sociodemográfico, de consumo y criminológico en la muestra.

\begin{tabular}{|c|c|c|}
\hline & $\%(\mathrm{~N})$ & $M(D T)$ \\
\hline \multicolumn{3}{|c|}{ Variables Sociodemográficas } \\
\hline \multicolumn{3}{|l|}{ Sexo } \\
\hline Hombre & $76.7 \%(135)$ & \\
\hline Mujer & $23.3 \%(41)$ & \\
\hline Edad & & $32.16(7.1)$ \\
\hline \multicolumn{3}{|l|}{ Convivencia } \\
\hline Solo & $10.8 \%(19)$ & \\
\hline Pareja & $10.8 \%(19)$ & \\
\hline Solo hijos & $1.7 \%(3)$ & \\
\hline Pareja + hijos & $17 \%(30)$ & \\
\hline Padres & $55.7 \%(98)$ & \\
\hline Amigos & $1.1 \%(2)$ & \\
\hline Otros & $2.8 \%(5)$ & \\
\hline \multicolumn{3}{|l|}{ Estado civil } \\
\hline Soltero & $74.4 \%(131)$ & \\
\hline Casado & $10.2 \%(18)$ & \\
\hline Viudo & $1.1 \%(2)$ & \\
\hline Divorciado/separado & $14.2 \%(25)$ & \\
\hline Sin Hijos & $71 \%(125)$ & \\
\hline \multicolumn{3}{|l|}{ Fuente ingresos } \\
\hline Laboral & $50 \%(88)$ & \\
\hline Ayuda familia & $23.8 \%(42)$ & \\
\hline Prestaciones & $17 \%(30)$ & \\
\hline Marginal & $7.4 \%(13)$ & \\
\hline \multicolumn{3}{|l|}{ Características del consumo } \\
\hline Edad Inicio & & $19.43(4.69)$ \\
\hline \multicolumn{3}{|l|}{ Frecuencia consumo } \\
\hline Todos los días & $60.2 \%(106)$ & \\
\hline 4-6 semana & $14.8 \%(26)$ & \\
\hline $2-3$ semana & $19.3 \%(34)$ & \\
\hline 1 semana & $2.8 \%(5)$ & \\
\hline Menos de 1 & $2.8 \%(5)$ & \\
\hline \multicolumn{3}{|l|}{ Droga Co-principal } \\
\hline Cocaína & $22.2 \%(39)$ & \\
\hline Alcohol & $44.3 \%(78)$ & \\
\hline Cannabis & $22.2 \%(39)$ & \\
\hline Benzodiacepina & $2.8 \%(5)$ & \\
\hline Tabaco & $5.7 \%(10)$ & \\
\hline \multicolumn{3}{|l|}{ Otras habitualmente } \\
\hline Heroína & $18.2(16)$ & \\
\hline Cocaína & $35.8 \%(63)$ & \\
\hline Alcohol & $77.8 \%(137)$ & \\
\hline Cannabis & $62.5 \%(110)$ & \\
\hline Benzodiacepina & $28.4 \%(50)$ & \\
\hline Drogas síntesis & $14.8 \%(26)$ & \\
\hline Tabaco & $94.3 \%(166)$ & \\
\hline № urgencias & & $.82(1.65)$ \\
\hline Tratamientos previos & $59.7 \%(105)$ & \\
\hline Tratamiento psiquiátrico & $48.3 \%(85)$ & \\
\hline Variables criminológicas & $34.7 \%(61)$ & \\
\hline Edad 10 delito & & $23.33(7.02)$ \\
\hline № detenciones & & $3.10(5.25)$ \\
\hline № meses prisión & & $3.70(10.40)$ \\
\hline Procesos judiciales & & $1.15(1.86)$ \\
\hline
\end{tabular}


Tabla 2. Correlaciones entre trastornos y variables de personalidad.

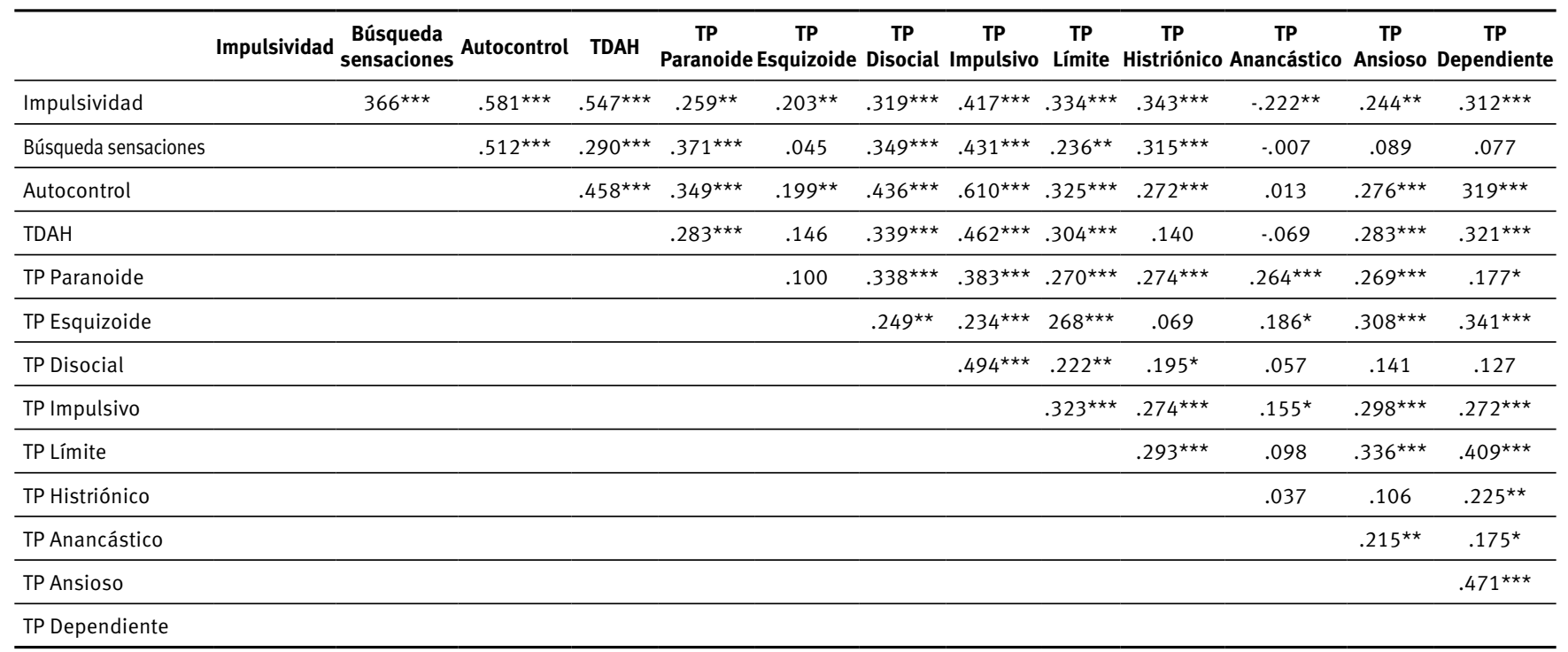

Nota: De acuerdo con las instrucciones de codificación de la escala de Grasmick et al. (1993), una alta puntuación en esta escala indica bajo autocontrol. Nota: ${ }^{\star} p<.05,{ }^{\star \star} p<.01,{ }^{\star \star \star} p<.001$

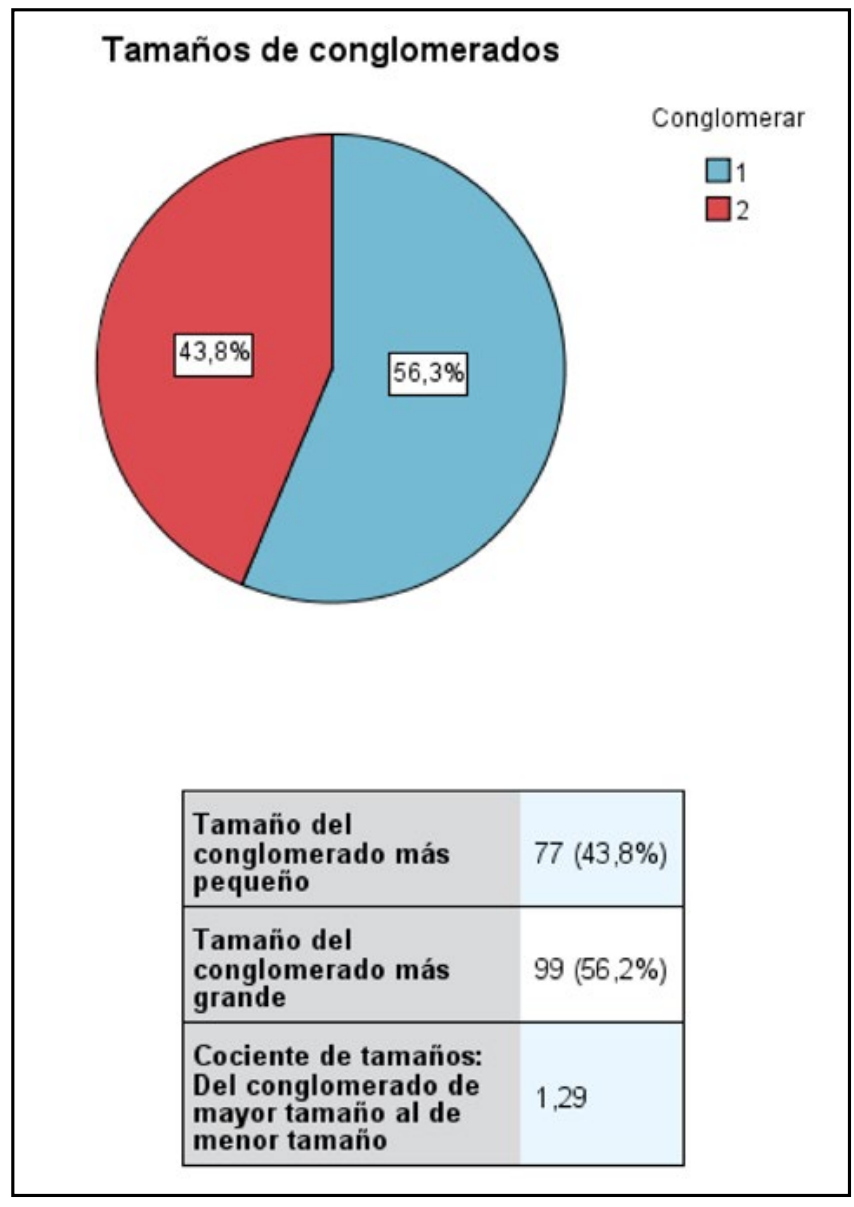

Figura 1. Tamaño de los conglomerados identificados en el análisis cluster.

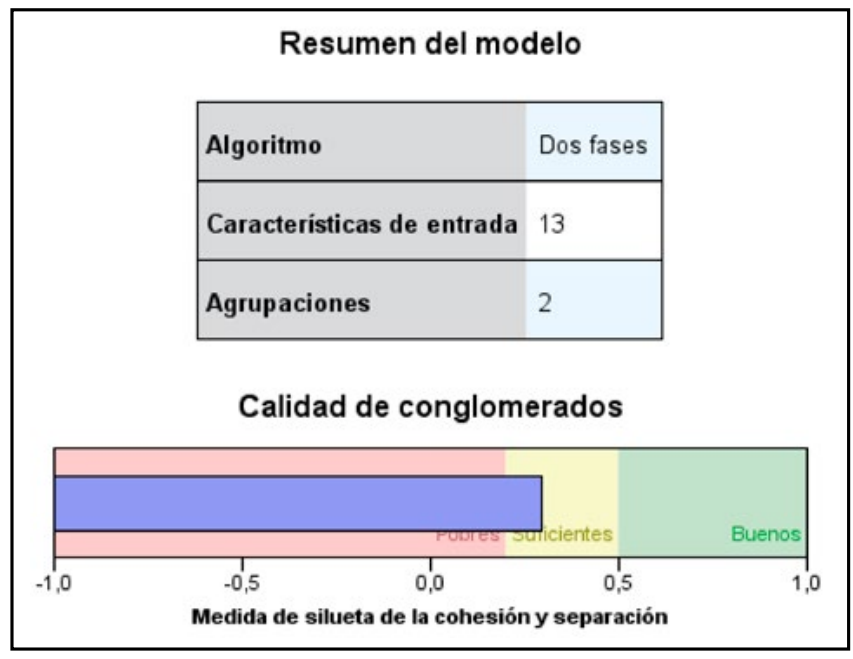

Figura 2. Resumen del modelo e índice de calidad de la solución identificada en el análisis cluster.

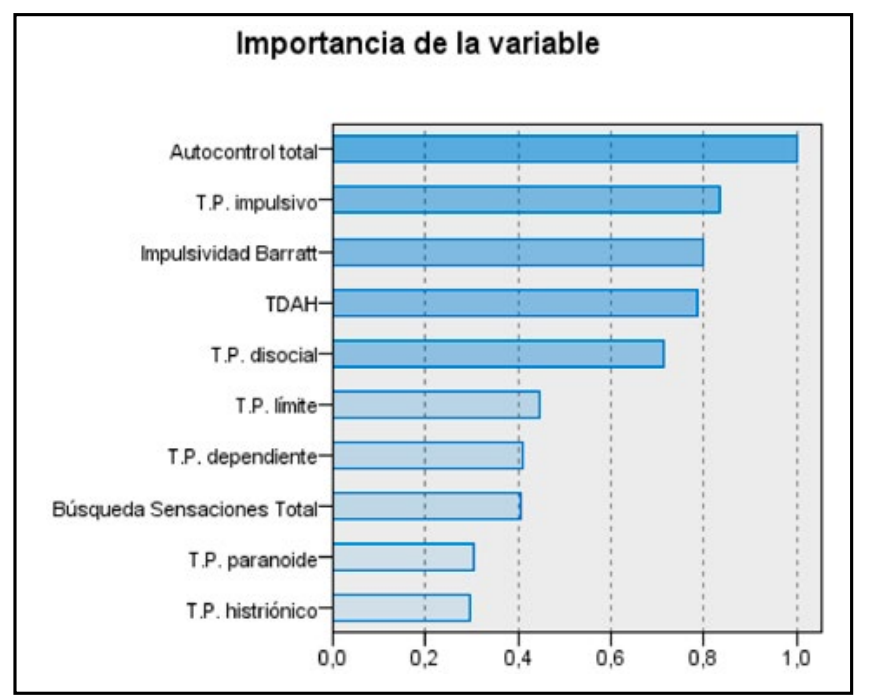

Figura 3. Importancia de las variables para diferenciar a los dos clusters. 
búsqueda de sensaciones tiene su correlación más alta con el TP impulsivo; finalmente, el autocontrol presenta sus más altas correlaciones con el TP impulsivo, TDAH y TP disocial.

\section{Perfiles de pacientes en relación a dimensiones de personalidad}

Para la identificación de perfiles homogéneos de adictos, se introdujeron en el análisis cluster las variables de personalidad/psicopatológicas mencionadas en la introducción que, consistentemente a lo largo de estudios previos, se mostraron relacionadas con la severidad del consumo de drogas: impulsividad, búsqueda de sensaciones, autocontrol, TDAH y TP. El análisis extrajo así una solución de dos clusters bien diferenciados, con $\mathrm{BIC}=1623.18$ (BIC para tres clusters $=$ 1645.00; BIC para cuatro clusters $=1719.10)$. Dado que la investigación previa tiende a identificar también dos subtipos, la solución de dos clusters fue la retenida para el estudio, tanto en virtud de criterios empíricos (BIC) como conceptuales. En la Figura 1 se muestra la composición de los dos clusters: un primer cluster con 77 participantes $(43.8 \%)$ y un segundo cluster con 99 participantes $(56.1 \%)$.

En las Figuras 2 y 3 se presentan, respectivamente, la prueba de calidad de conglomerados y las variables con mayor peso en la agrupación de los clusters.
Las variables que más contribuyen a definir los grupos fueron: autocontrol, TP impulsivo, impulsividad, TDAH y TP disocial, tal y como se presenta en la Figura 3.

\section{Diferencias de los perfiles en variables de personali- dad y trastornos}

Con el fin de tener una caracterización más precisa de los clusters en función de las variables introducidas para su definición, los grupos fueron comparados en las variables de personalidad y trastornos. Esto permitirá detallar qué perfiles de personalidad emergen con cada uno de los grupos. Dado que se realizan múltiples comparaciones en variables relacionadas entre sí, se utilizó un MANOVA. En la Tabla 3 se presentan los resultados.

Como se observa en la tabla, los participantes del cluster 2 puntúan significativamente más alto en todas las variables ( $\mathrm{p}<.003$, considerando el ajuste de Bonferroni para el control del error tipo I), salvo en el TP anancástico. Los individuos que componen el cluster 2 parecen tener un perfil más severo en términos de bajo autocontrol, elevada impulsividad, elevada búsqueda de sensaciones y mayor puntuación en el TDAH y en la mayoría de los TP.

\section{Asociación entre los perfiles y los grupos de consumo}

Además, con el fin de conocer cómo los tres grupos de consumo considerados en este estudio (heroinómanos, co-

Tabla 3. Resultados del análisis multivariable de la varianza para la comparación de los dos clusters en las variables de personalidad y trastornos.

\begin{tabular}{|c|c|c|c|c|c|c|c|c|}
\hline & & Grupo 1 & & & & & & \\
\hline & $M$ & DT & $M$ & DT & $\Lambda$ & $F(g l)$ & Sig. & $\eta^{2}$ \\
\hline & & & & & .35 & $22.61(13,161)$ & $<.001$ & .65 \\
\hline Impulsividad & 54.34 & 12.58 & 73.22 & 13.52 & & $81.21(1,173)$ & $<.001$ & .31 \\
\hline Búsqueda sensaciones & 20.63 & 5.31 & 25.77 & 5.45 & & $31.53(1,173)$ & $<.001$ & .15 \\
\hline Autocontrol & 10.65 & 3.78 & 16.88 & 3.60 & & $112.91(1,173)$ & $<.001$ & .39 \\
\hline TDAH & 10.05 & 3.06 & 14.72 & 3.50 & & $75.71(1,173)$ & $<.001$ & .30 \\
\hline TP Paranoide & 3.31 & 1.35 & 4.36 & 1.25 & & $22.60(1.173)$ & $<.001$ & .11 \\
\hline TP Esquizoide & 2.85 & 1.67 & 4.00 & 1.77 & & $20.10(1,173)$ & $<.001$ & .10 \\
\hline TP Disocial & 1.57 & 1.19 & 3.23 & 1.26 & & $69.19(1,173)$ & $<.001$ & .28 \\
\hline TP Impulsivo & 1.83 & 1.26 & 3.53 & 0.94 & & $79.14(1,173)$ & $<.001$ & .31 \\
\hline TP Límite & 2.32 & 1.20 & 3.46 & 1.03 & & $39.06(1,173)$ & $<.001$ & .18 \\
\hline TP Histriónico & 2.07 & 1.29 & 3.07 & 1.25 & & $20.97(1,173)$ & $<.001$ & .18 \\
\hline TP Anancástico & 2.98 & 1.65 & 3.10 & 1.58 & & $.82(1,173)$ & .351 & .01 \\
\hline TP Ansioso & 3.33 & 1.40 & 4.28 & 1.21 & & $18.94(1,173)$ & $<.001$ & .09 \\
\hline TP Dependiente & 2.14 & 1.34 & 3.41 & 1.30 & & $38.01(1,173)$ & $<.001$ & .18 \\
\hline
\end{tabular}

Tabla 4. Resultados del análisis de chi-cuadrado entre los dos clusters y los tres grupos de consumidores.

\begin{tabular}{lccccc}
\hline & Cocaina & Heroina & Cocaheroína & Total & Chi $^{2}$ \\
\hline Grupo 1 & $58.3 \%(42)$ & $60.2 \%(50)$ & $25.0 \%(4)$ & $56.1 \%(96)$ & 7.00 \\
\hline Grupo 2 & $41.7 \%(30)$ & $39.8 \%(33)$ & $75.0 \%(12)$ & $43.9 \%(75)$ & \\
\hline
\end{tabular}


Tabla 5. Resultados de los análisis multivariables de la varianza para la comparación de los clusters en los indicadores de gravedad en el consumo e indicadores criminológicos.

\begin{tabular}{|c|c|c|c|c|c|c|c|c|}
\hline & \multicolumn{2}{|c|}{ Grupo 1} & \multicolumn{2}{|c|}{ Grupo 2} & \multirow[b]{2}{*}{$\Lambda$} & \multirow[b]{2}{*}{$F(g l)$} & \multirow[b]{2}{*}{ Sig. } & \multirow[b]{2}{*}{$\eta^{2}$} \\
\hline & $\mathbf{M}$ & DT & $\mathbf{M}$ & DT & & & & \\
\hline Indicadores gravedad consumo & & & & & .42 & $4.78(1,167)$ & $<.001$ & .19 \\
\hline Edad inicio consumo & 20.55 & 5.16 & 17.99 & 3.55 & & $7.41(1,173)$ & $<.05$ & .10 \\
\hline Frecuencia consumo & 1.68 & 1.03 & 1.81 & 1.06 & & $.05(1,173)$ & .82 & .00 \\
\hline № urgencias & .59 & 1.16 & 1.10 & 2.10 & & $6.23(1,173)$ & $<.001$ & .10 \\
\hline Tratamientos previos & .59 & .49 & .61 & .49 & & $3.30(1,173)$ & $<.05$ & .03 \\
\hline Indicadores criminológicos & & & & & .38 & $4.49(4,56)$ & $<.01$ & .16 \\
\hline № detenciones & .65 & 1.30 & 1.67 & 4.93 & & $3.21(1,59)$ & $<.05$ & .07 \\
\hline Edad primer delito & 24.73 & 7.40 & 21.97 & 6.45 & & $2.42(1,59)$ & .12 & .03 \\
\hline Meses prisión & 1.62 & 7.97 & .92 & 3.35 & & $1.16(1,59)$ & .28 & .02 \\
\hline Procesos judiciales & .51 & .97 & 1.13 & 2.44 & & $5.23(1,59)$ & $<.01$ & .10 \\
\hline
\end{tabular}

cainómanos y cocaheroinómanos) se distribuyen entre los dos clusters, se realizó un análisis de tablas de contingencia, tal y como se presenta en la Tabla 4.

Como se observa en la tabla, chi-cuadrado fue estadísticamente significativo, indicando una desigual distribución de los individuos entre los grupos. Particularmente, se observa que una elevada proporción de cocaheroinómanos se concentra en el cluster de personalidad más severo (cluster 2). Concretamente, el 75\% de los cocaheroinómanos pertenecerían al cluster 2, frente al $25 \%$ que formarían parte del cluster 1 . De los cocainómanos, el $41.7 \%$ está en el cluster 2, y el $39.8 \%$ de los heroinómanos se sitúan en este cluster 2 .

Además, ambos grupos fueron comparados en sexo y edad. No se encontraron diferencias en sexo, con chi-cuadrado $(1 \mathrm{gl})=2.13, \mathrm{p}=.155$, pero sí en edad, $\mathrm{F}(1,174)=$ 12.44, $\mathrm{p}<.001$, con mayor edad en el cluster $1(\mathrm{M}=33.78$, DT $=7.06)$ que en el cluster $2(\mathrm{M}=30.09, \mathrm{DT}=6.63)$.

\section{Perfiles en relación con indicadores de gravedad de consumo y criminológico}

Finalmente, se examinó cómo los clusters se asocian con indicadores de severidad en el consumo e indicadores criminológicos evaluados en este estudio. Dado que los grupos difieren en edad, y esta pudiera ser una variable espuria, afectando a los resultados de las comparaciones, los análisis se realizaron controlando la variable edad en los MANOVA. Los resultados de los análisis se presentan en la Tabla 5.

Tal y como se observa en la tabla, la edad de inicio del consumo es significativamente menor en el cluster 2 , si bien esta significación se convierte en limítrofe al aplicar la corrección de Bonferroni $(\mathrm{p}<.012)$. Tras aplicar esta corrección, sin embargo, se mantiene significativa la diferencia en el número de urgencias, mayor en el cluster 2. Se observa una tendencia a un mayor número de tratamientos previos en el cluster 2 ( $\mathrm{p}=$ $.05)$, no significativa después del ajuste Bonferroni.
Con respecto a los indicadores criminológicos, la cantidad de procesos judiciales en los que han estado implicados los participantes son mayores en el cluster 2 , con una diferencia que permanece significativa después de la corrección de Bonferroni $(\mathrm{p}<.012)$; el número de detenciones también tiende a ser, con un nivel de significación marginal, mayor en el cluster 2 .

\section{Discusión}

Este trabajo tiene el propósito de ahondar en la heterogeneidad de la población de adictos, examinando si, en ellos, pueden identificarse perfiles personales diferenciados. Los resultados del análisis cluster revelaron datos de interés con respecto a las tipologías de los adictos a sustancias, delimitando dos grupos diferenciados especialmente en las variables autocontrol, TP impulsivo, impulsividad total, TDAH y TP disocial. El segundo agrupa a los que puntúan significativamente más alto en prácticamente todas las variables y trastornos estudiados y obtiene un perfil personal que sugiere una mayor desinhibición, y por tanto más riesgo de gravedad, mostrando menor autocontrol, elevada impulsividad y búsqueda de sensaciones, y mayores puntuaciones en los trastornos, tanto de personalidad como de TDAH. Este cluster además incluye a los pacientes con indicadores de gravedad y criminológicos que, en general, de acuerdo con los MANOVA, son más severos, particularmente con más urgencias por uso de sustancias y más procesos judiciales, lo que corrobora las dificultades psicosociales sugeridas por su patrón de personalidad.

Estos resultados son comparables con los subtipos de adicción al alcohol que encontró Cloninger (1987): el tipo I con bajas puntuaciones en búsqueda de novedad, y el tipo II con elevadas puntuaciones en búsqueda de novedad, inicio temprano de los problemas con el alcohol y consumo más continuado, entre otras características; o los denominados 
por Babor et al. (1992) tipo A y B. El perfil I o A sería el menos grave, con inicio más tardío, menor heredabilidad y factores de riesgo en infancia, y menor dependencia, mientras que el II o B tendría más gravedad, dependencia, heredabilidad, búsqueda de novedad y sensaciones, conducta impulsiva o antisocial. Ball (1995) encuentra resultados similares en un análisis sobre consumidores de cocaína, siendo los del grupo B los que presentan más factores de riesgo comórbido, severidad en el abuso de cocaína y alcohol, más problemática psicosocial relacionada, TP antisocial, problemas psiquiátricos, más criminalidad y más tratamientos por drogas y psiquiátricos, así como mayores puntuaciones en búsqueda de sensaciones. Así nos encontramos con que los perfiles diferenciados que aparecen en nuestro estudio se corresponderían a los citados anteriormente, el II o B más grave que el tipo I o A. Del mismo modo, se encuentra cierto paralelismo con las llamadas por Muñoz et al. (2006) tipología funcional y crónica, esta última con mayor gravedad, más años de consumo y problemas psicosociales asociados a las drogas. La distinción que encontramos en nuestro estudio podría corresponderse también con los tipos identificados por Echeburúa, Bravo de Medina y Aizpiri (2008), quienes en un estudio con alcohólicos encuentran que los de tipo II además de mayor impulsividad y búsqueda de sensaciones, también presentan más TP (en concreto los trastornos obsesivo, narcisista, paranoide y antisocial), más trastornos dramáticos/erráticos (TP antisocial, límite, histriónico y narcisista); también que en los alcohólicos de tipo II aparece un perfil de más sintomatología psicopatológica (impulsividad y hostilidad), por lo que se recomendaría programas de tratamiento multicomponentes. También nuestros resultados son coherentes con los hallazgos de Graña et al. (2006) en el sentido de mostrar que las puntuaciones más altas en el trastorno disocial son una característica de los drogodependientes crónicos.

Así pues, los clusters encontrados son similares a estudios precedentes tanto en alcoholismo como en sustancias ilegales, apareciendo una tipología 2 con un perfil más severo, y que se corresponde principalmente a los pacientes cocaheroinómanos, que en anteriores trabajos (Carou et al., 2013) se había encontrado que tenían un perfil de mayor gravedad, y marginalidad, y también de problemas legales, con inicio más temprano en el consumo, mayor impulsividad y susceptibilidad al aburrimiento. Estos resultados, además, ratifican la delineación de los pacientes cocaheroinómanos como una categoría especial dentro de los consumidores de cocaína, con un perfil propio, que merece ser considerado de un modo específico en el ámbito del tratamiento de las drogodependencias. Otros estudios, de hecho, han observado que, entre los consumidores, los que son adictos a más de una sustancia son más impulsivos que los que lo son a una sola (McCown, 1988; O’Boyle y Barratt, 1993). En este sentido, Farrington (1992) ha señalado la existencia una predisposición general de tendencia antisocial cons- tituida por una serie de factores de riesgo entre los que destacan la impulsividad, la hiperactividad, la búsqueda de sensaciones, la toma de riesgos y la incapacidad para demorar la gratificación.

Por otra parte, se confirman los resultados de otros estudios que ponen en relación la alta búsqueda de sensaciones y la impulsividad con la adicción (Ball, 2004; Hittner y Swickert, 2006) y también en gravedad de la adicción (Dom, De Wilde, Hulstijn, Van Den Brink, y Sabbe, 2006; Horvath, Milich, Lynam, Leukefeld, y Clayton, 2004). Parece existir una asociación entre trastornos que tienen en común la impulsividad, destacando los trastornos de control de impulsos, los de personalidad, los relacionados con uso de sustancias, y el TDAH, dando lugar a comorbilidad e incluso lo que comienza a llamarse "trimorbilidad" (Tiffon, 2008). La aparición de TP (y en concreto de trastornos disocial, impulsivo, límite) así como de TDAH se relaciona en la literatura con la presencia de adicciones, sirviendo como indicadores de gravedad en el consumo (Ball, 1995), tanto con el inicio a una más temprana edad en el consumo, como con la presencia de más urgencias o con el fracaso en los tratamientos (Verheul et al., 1995, Biederman, Wilens, Mick, Faraone, y Spencer, 1998). Esto es algo que se ratifica también en nuestro estudio.

Este trabajo presenta limitaciones que deben de ser tenidas en cuenta. Así, se ha analizado una muestra de pacientes que no ha podido ser comparada con un grupo control de población general, ni la recogida de datos se ha podido ampliar a diferentes centros lo que permitiría considerar otros grupos de consumidores; en segundo lugar, la evaluación de las variables de personalidad y trastornos se ha realizado exclusivamente a través de cuestionarios, lo cual está sujeto a sesgos detectados en la literatura previa, como la deseabilidad social; en tercer lugar, han quedado fuera del estudio otras sustancias importantes por las que las personas pueden acudir a tratamiento, como el cannabis o el alcohol, de manera que estos resultados no pueden generalizarse a las drogodependencias, en general, sino ser contextualizados con respecto a las sustancias estudiadas y en individuos que han solicitado ayuda para ello; finalmente, la naturaleza transversal de este estudio no permite conocer cómo las características de personalidad y los trastornos predicen, prospectivamente, el ajuste de los adictos ni su respuesta al tratamiento. Pese a ello, se ha podido comprobar la existencia de perfiles específicos en distintos tipos de consumidores, relacionados con variables de personalidad. El estudio ha permitido constatar también la importancia de los TP y el TDAH en la delimitación de un subtipo con características más severas de consumo. Los trastornos ligados a la impulsividad y la desinhibición parecen desempeñar un papel crucial en la caracterización psicológica de los patrones de adicción más severa, y esto deberá ser considerado a la hora de valorar las necesidades terapéuticas de los adictos. 


\section{Conflicto de intereses}

Los autores declaran que no existe ningún conflicto de interés en este trabajo.

\section{Referencias}

Babor, T. F., Hofmann, M., Desboca, F. K., Hesselbrock, V., Meyer, R. E., Dolinski, Z. S. y Rounsaville, B. (1992). Types of alcoholics, I: Evidence for an empirically derived typology based on indicators of vulnerability and severity. Archives of General Psychiatry, 49, 599-608. doi:10.1001/archpsyc.1992.01820080007002.

Ball, S. A. (1995). The validity of an alternative five-factor measure of personality in cocaine abusers. Psychological Assessment, 7, 148-154. doi:10.1037/1040-3590.7.2.148.

Ball, S. A. (2004). Personality traits, disorders and substance abuse. En R. M. Stelmack (Ed.), On the psychobiology of personality: Essays in honor of Marvin Zuckerman (pp. 203-222). Amsterdam: Elsevier. doi:10.1016/B978008044209-9/50013-0.

Belin, D., Mar, A. C., Dalley, J. W., Robbins, T. W. y Everitt, B. J. (2008). High impulsivity predicts the switch to compulsive cocaine-taking. Science, 320, 1352-1355. doi:10.1126/science.1158136.

Bergman, L. R. y Magnusson, D. (1997). A person-oriented approach in research on developmental psychopathology. Development and Psychopathology, 9, 291-319. doi:10.1017/S095457949700206X.

Biederman, J., Wilens, T. E., Mick, E., Faraone, S. V. y Spencer, T. (1998). Does attention-deficit hyperactivity disorder impact the developmental course of drug and alcohol abuse and dependence? Biological Psychiatry, 44, 269-273. doi:10.1016/S0006-3223(97)00406-X.

Biederman, J., Wilens, T. E., Mick, E., Milberger, S., Spencer, T. J. y Faraone, S. V. (1995). Psychoactive substance use disorders in adults with attention-deficit/hyperactivity disorder (ADHD): Effects of ADHD and psychiatric comorbidity. American Journal of Psychiatry, 152, 16521658.

Carou, M., Romero, E. y Luengo, M. A. (2013). Patrones de consumo y variables de personalidad en drogodependientes a tratamiento. Revista Española de Drogodependencias, 3, 217-232.

Carrillo-de-la-Peña, M. T., Otero, J. M. y Romero, E. (1993). Comparison among various methods of assessment of impulsiveness. Perceptual and Motor Skills, 77, 567-575.

Cloninger, C. R. (1987). A systematic method for clinical description and classification of personality variants. Archives of General Psychiatry, 44, 573-588. doi:10.1001/ archpsyc.1987.01800180093014.

Daigre, C., Ramos-Quiroga, J. A., Valero, S., Bosch, R., Roncero, C., Gonzalvo, B. y Nogueira M. (2009). Adult ADHD Self-Report Scale (ASRS-v1.1) symptom checklist in patients with substance use disorders. Actas Españolas de Psiquiatría, 37, 299-305.

Dom, G., De Wilde, B., Hulstijn, W., Van Den Brink, W. y Sabbe, B. (2006). Behavioral aspects of impulsivity in alcoholics with and without a cluster-B personality disorder. Alcohol and Alcoholism, 41, 412-420.

Echeburúa, E., Bravo De Medina, R. y Aizpiri, J. (2008). Variables de personalidad, alteraciones psicopatológicas y trastornos de personalidad en pacientes con dependencia de alcohol en función de la tipología de Cloninger. Psicothema, 20(4), 525-530.

Echeburúa, E., De Medina, R. B. y Aizpiri, J. (2007). Comorbidity of alcohol dependence and personality disorders: A comparative study. Alcohol and Alcoholism, 42(6), 618-622. doi:10.1093/alcalc/agm050.

Farrington, D. P. (1992). Explaining the beginning, progress and ending of antisocial behaviour from birth to adulthood. En J McCord (Eds.), Facts, frameworks and forecasts: Advances in criminological theory, 3 (pp. 353-286). New Brunswick, NJ: Transaction Press.

Fillmore, M. T., Ostling, E. W., Martin, C. A. y Kelly, T. H. (2009). Acute effects of alcohol on inhibitory control and information processing in high and low sensation-seekers. Drug and Alcohol Dependence, 100, 91-99. doi:10.1016/j.drugalcdep.2008.09.007.

Frei, A. y Rehm, J. (2002). The prevalence of psychiatric co-morbidity among opioid addicts. Psychiatrische Praxis, 29, 258-262.

Gallupe, O. y Baron, S. W. (2014). Morality, self-control, deterrence, and drug use: Street youths and situational action theory. Crime EO Delinquency. 60, 284-305. doi:10.1177/0011128709359661.

González, C. (2014). Screening for personality disorder in drug and alcohol dependence. Psychiatry Research, 217, 121-123. doi:10.1016/j.psychres.2014.03.007.

Gottfredson, M. R. y Hirschi, T. (1990). A general theory of crime. Stanford, CA: Stanford University Press

Gottfredson, M. R. y Hirschi, T. (1994). A general theory of adolescent problem behavior. En R. D. Ketterlinus y M. E. Lamb (Eds), Adolescent problem behaviors: Issues and research (pp. 41-56). Hillsdale, NY: Erlbaum.

Graña, J. L., Muñoz, J. J. y Navas, E. (2009). Normal and pathological personality characteristics in subtypes of drug addicts undergoing treatment. Personality and Individual Differences, 46, 418-423.

Grasmick, H. G., Title, C. R., Bursik, R. J. y Arneklev, B. J. (1993). Testing the core empirical implications of Gottfredson and Hirschi's general theory of crime. Journal of Research in Crime and Delinquency, 30, 5-29.

Grogan, K. y Bramham, J. (2016). Demographic, developmental and psychosocial predictors of the development of anxiety in adults with ADHD. Journal of Attention Deficit and Hyperactivity Disorder, 8, 35-44. doi:10.1007/s12402-015-0183-0. 
Gullo, M. J., Loxton, N. J. y Dawe, S. (2014). Impulsivity: Four ways five factors are not basic to addiction. Addictive Behaviors. 39, 1547-1556. doi:10.1016/j.addbeh.2014.01.002.

Hittner, J. B. y Swickert, R. (2006) Sensation seeking and alcohol use: A meta-analytic review. Addictive Behaviors, 31, 1383-1401. doi:10.1016/j.addbeh.2005.11.004.

Horvath, L. S., Milich, R., Lynam, D., Leukefeld, C. y Clayton, R. (2004). Sensation Seeking and substance use: A cross-lagged panel design. Individual Differences Research, 2, 173-183.

Kalbag, A. S. y Levin, F. R. (2005). Adult ADHD and substance abuse: Diagnostic and treatment issues. Substance Use and Misuse, 40, 1955-1981. doi:10.1080/10826080500294858.

Kessler, R. C., Adler, L., Ames, M., Delmer, O., Faraone, S., Hiripi, E.,... Walters E.E. (2005). The World Health Organization Adult ADHD Self-Report Scale (ASRS): A short screening scale for use in the general population. Psychological Medicine, 35, 245-256. doi:10.1017/ S0033291704002892.

Kessler, R. C., Adler, L. A., Gruber, M. J., Sarawate, C. A., Spencer, T. y Van Brunt, D. L. (2007). Validity of the World Health Organization Adult ADHD Self-Report Scale (ASRS) screener in a representative sample of health plan members. International Journal of Methods in Psychiatric Research, 16, 52-65. doi:10.1002/mpr.208.

López-Ibor, J. J., Pérez-Urdaniz, A. y Rubio-Larrosa, V. (1996). Examen Internacional de los Trastornos de la Personalidad (IPDE) Módulo DSM-IV y CIE-10. Madrid: Meditor.

López-Torrecillas, F., Peralta, I., Muñoz-Rivas, M. J. y Godoy, J. F. (2003). Autocontrol y consumo de drogas. Adicciones, 15, 127-136. doi:10.20882/adicciones. 436.

López, A. y Becoña, E. (2006). Consumo de cocaína y psicopatología asociada: una revisión. Adicciones, 18, 161-196.

Luengo, A., Otero-López, J. M., Romero, E. y Gómez, J. A. (1996). Efectos de la necesidad de búsqueda de sensaciones sobre la involucración en el consumo de drogas de los adolescentes. Análisis y Modificación de Conducta, 22, 683-708.

Magnavita, J.J. (Ed.) (2004). Handbook of personality disorders: Theory and practice. Nueva York: Wiley.

McCown, W. G. (1988). Multi-impulsive personality disorder and multiple substance abuse: Evidence from members of self-help groups. British Journal of Addiction, 83, 431-432. doi:10.1111/j.1360-0443.1988.tb00490.x.

Motos-Sellés, P., Cortés-Tomás, M.T., Giménez-Costa, J.A. y Cadaveira-Mahía, F. (2015). Predictores del consumo semanal de alcohol y sus consecuencias asociadas en universitarios consumidores intensivos de alcohol. Adicciones, 27, 119-131. doi:10.20882/adicciones.700.

Muñoz, J. J., Navas, E., Graña, J. L. y Martínez, R. (2006). Subtipos de drogodependientes en tratamiento: apoyo empírico para una distinción entre Tipo A y Tipo B. Psicothema, 18(1), 43-51.
Nadal-Alemany, R. (2008). La búsqueda de sensaciones y su relación con la vulnerabilidad a la adicción y al estrés. Adicciones, 20, 59-72. doi:10.20882/adicciones.289.

O'Boyle, M. y Barratt, E. S. (1993). Impulsivity and DSM-III-R personality disorders. Personality and Individual Differences, 14, 609-611. doi:10.1016/01918869(93)90156-W.

Olmstead, M. C. (2006). Animal models of drug addiction: Where do we go from here? Quarterly Journal of Experimental Psychology, 59, 625-653. doi:10.1080/17470210500356308.

Oquendo, M. A., Baca-García, E., Graver, R., Morales, M., Montalvan, V. y Mann, J. J. (2001). Spanish adaptation of the Barratt Impulsiveness Scale (BIS-11). European Journal of Psychiatry, 15, 147-155.

Patton, J. H., Stanford, M. S. y Barratt, E. S. (1995). Factor structure of the Barratt Impulsiveness Scale. Journal of Clinical Psychology, 51, 768-774.

Pérez, J. y Torrubia, R. (1986). Fiabilidad y validez de la versión española de la escala de Búsqueda de Sensaciones-Forma V. Revista Latino-americana de Psicología, 18, 7-22.

Ramos-Quiroga, J. A., Bosch-Munsó, R., Castells-Cervelló, X., Nogueira-Morais, M., García-Giménez, E. y Casas-Brugué, M. (2006). Trastorno por déficit de atención con hiperactividad en adultos: caracterización clínica y terapéutica. Revista de Neurología, 42, 600-606.

Romero, E. y Alonso, C. (2015). Hyperactive behaviors from childhood to adolescence: Prospective outcomes in a sample of Spanish children. International Journal of Psychological Studies, 7, 67-75. doi:10.5539/ijps.v7n3p67.

Romero, E., Gómez-Fraguela, J. A., Luengo, M. A. y Sobral, J. (2003). The self-control construct in the general theory of crime: An investigation in terms of personality psychology. Psychology, Crime and Law, 9, 61-86. doi:10.1080/10683160308142.

Romero, E., Luengo, M. A. y Sobral, J. (2001). Personality and antisocial behaviour: Study of temperamental dimensions. Personality and Individual Differences, 31, 329348.

Romero, E., Sobral, J. y Luengo, M. A. (1999). Personalidad y delincuencia: Entre la biología y la sociedad. Granada, España: Grupo Editorial Universitario.

Romero-Martínez, A. y Moya-Albiol, L. (2015). Déficits neuropsicológicos asociados a la relación entre abuso de cocaína y violencia: Mecanismos neuronales facilitadores. Adicciones, 27, 64-74. doi:10.20882/adicciones.194.

Schubiner, H., Tzelepis, A., Milberger, S., Lockhart, N., Kruger, M., Kelley, B. J. y Schoener, E. P. (2000). Prevalence of attention-deficit/hyperactivity disorder and conduct disorder among substance abusers. Journal of Clinical Psychiatry, 61, 244-251. doi:10.4088/JCP.v61n0402.

Slade, K. y Forrester, A. (2013). Measuring IPDE-SQ personality disorder prevalence in presentence and ear- 
ly-stage prison populations, with sub-type estimates. International Journal of Law and Psychiatry, 36, 207-212.

Stautz, K. y Cooper, A. (2013). Impulsivity-related personality traits and adolescent alcohol use: A meta-analytic review. Clinical Psychology Review, 33(4), 574-592. doi:10.1016/j.cpr.2013.03.003.

Tiffon, B. N. (2008). Una trimorbilidad forense emergente: el trastorno de personalidad, el trastorno del control de los impulsos y el abuso de sustancias tóxicas. Anuario de Psicología Jurídica, 18, 91-97.

Verheul, R. (2001). Co-morbidity of personality disorder in individuals with substance use disorders. European Psychiatry, 16, 274-282. . doi:10.1016/S0924-9338(01)00578-8.

Verheul, R., van der Brink, W. y Hartgers, C. (1995). Prevalence of personality disorders among alcoholics and drug addicts: An overview. European Addiction Research, 1, 166-177. doi:10.1016/S0924-9338(01)00578-8.

Wilens T. E. (2007). The nature of the relationship between attention-deficity/hyperactivity disorder and substance abuse. Journal of Clinical Psychiatry, 68, 4-8.

Zuckerman, M. (1979). Sensation Seeking: Beyond the optimal level of arousal. NY: Erlbaum.

Zuckerman, M., Eysenck, S. y Eysenck, H. J. (1978). Sensation seeking in England and America: Cross-cultural, age and sex comparisons. Journal of Consulting and Clinical Psychology, 46, 139-149. doi:10.1037/0022006X.46.1.139. 